\title{
Analgesic Effect of a 5\% Lidocaine Patch in Patients With Opioid Treatment-Resistant Neuropathic Cancer Pain
}

\section{Jui-hung Tsai}

National Cheng Kung University College of Medicine https://orcid.org/0000-0003-0318-3474

I-Ting Liu

National Cheng Kung University Hospital

\section{Pei-Fang Su}

National Cheng Kung University

\section{Ying-Tzu Huang}

National Cheng Kung University Hospital

\section{Ge-Lin Chiu}

National Cheng Kung University Hospital

\section{Yu-Yeh Chen}

National Cheng Kung University Hospital

\section{Wei-Shu Lai}

National Cheng Kung University College of Medicine

Peng-Chan Lin ( $\nabla$ pengchan@mail.ncku.edu.tw)

National Cheng Kung University College of Medicine

\section{Research Article}

Keywords: Neuropathic pain, Transdermal patch, Cancer pain, Visual analog scale, Analgesic

Posted Date: June 28th, 2021

DOl: https://doi.org/10.21203/rs.3.rs-564703/v1

License: (c) (1) This work is licensed under a Creative Commons Attribution 4.0 International License. Read Full License 


\section{Abstract}

\section{Purpose}

Limited efficacy has been observed when using morphine to treat neuropathic pain. Lidocaine patches reduce neuropathic pain in post-herpetic neuralgia, but their benefits for cancer-related neuropathic pain remain unclear. This study aimed to demonstrate a useful treatment for cancer-related neuropathic pain. The primary endpoint was pain intensity evaluated by the visual analog scale (VAS). The secondary endpoints were the pain relief score and the quality of analgesic treatment.

\section{Methods}

We assessed the efficacy and safety of lidocaine patches in patients experiencing neuropathic cancer pain. Terminal cancer patients with opioid treatment resistance participated in the 3-day study.

\section{Results}

The results showed a statistically significant difference in the median VAS over three days (Kruskal-Wallis test, $\mathrm{P}<0.0001)$. The median VAS pain intensity from Day 1 to Day 3 was 4.0 with $95 \%$ C.I. $(3.3,5.0), 3.0$ $(2.5,3.5)$ and $2.5(2.0,3.0)$, respectively. The difference between the median VAS pain intensities of any two days was statistically significant (Wilcoxon signed-rank test, $P<0.0001$ ). There was no statistically significant difference in the pain relief score or the quality of analgesic treatment.

Conclusion

In this study, the $5 \%$ lidocaine transdermal patch reduced the VAS pain intensity in neuropathic cancer patients with morphine resistance. The transdermal patch is generally useful and well-tolerated.

\section{Background}

According to the World Health Organization (WHO) guidelines, cancer pain treatment is effective and safe [1]. Neuropathic pain (NP) is a common problem encountered in $40 \%$ of cancer patients [2,3]. NP that results from neoplastic insults to the peripheral or central nervous system is characterized by continuous or intermittent spontaneous pain and abnormal sensitivity of the painful site to a variety of noxious (hyperalgesic) or innocuous (allodynic) stimuli [4,5]. Evoked pain that results from light touch by garments, running water or even cold air can be too bothersome for many of these patients. However, neuropathic pain is often described as 'intractable' and 'opioid resistant,' and the efficacy of opioids, nonsteroidal anti-inflammatory drugs, and other adjuvants remains controversial [6].

Several classes of drugs are moderately effective, but complete or near-complete relief is unlikely. Antidepressants and anticonvulsants are most commonly used. Opioid analgesics can provide some relief but are less effective for NP than for nociceptive pain; adverse effects may prevent adequate analgesia. Topical drugs and a lidocaine-containing patch may be useful for peripheral syndromes [7]. 
Sympathetic blockade is usually ineffective, except for some complex regional pain syndromes [8]. A lidocaine patch may be useful in relieving numbness, tingling, and other symptoms of neuropathy [9]. It is unknown whether a lidocaine patch is effective in treating neuropathy in cancer patients whose pain is refractory to morphine.

Lidocaine is an aminoethylamide local anesthetic that blocks voltage-gated sodium channels on excitable membranes, thereby preventing the generation and conduction of nerve impulses and providing analgesic relief $[10,11]$. The systemic absorption of lidocaine from the patch is minimal. The amount absorbed is directly related to the skin surface area covered and the lidocaine patch application duration. A $5 \%$ lidocaine patch was approved by the FDA for relief of pain associated with post-herpetic neuralgia and was marketed in March 1999 in the United States. The patch helps provide relief at the site of pain caused by damaged nerves. This new formulation of topical lidocaine is applied to intact painful skin and has been shown to produce significant pain relief without clinically significant serum levels $[10,11]$.

The present study aimed to evaluate the analgesic effect of a $5 \%$ lidocaine patch in patients with neuropathic cancer pain exhibiting opioid treatment resistance, and neuropathic pain was assessed by pain intensity via the visual analog scale (VAS), pain relief score, and analgesic treatment quality [12-14]. We evaluated the efficacy and safety of lidocaine patches in patients experiencing cancer-related neuropathic pain.

\section{Patients And Methods}

\section{Demographic characteristics}

A total of 96 terminal cancer patients, all with stage IV malignant disease and been admitted to hospice ward at National Cheng-Kung University Hospital (NCKUH) were enrolled. The eligibility criteria were 18 years of age and above; male or female; and experiencing neuropathic cancer pain evaluated by the LANSS Pain Scale [15] with opioid treatment resistance and without contraindication to topical anesthetic application. The study period was 3 days, and all participants signed informed consent forms. The symptoms of patients were evaluated by a questionnaire. The exclusion criteria were skin lesions with bacterial infection; history of allergies to para-aminobenzoic acid derivative drugs (procaine, tetracaine, benzocaine, etc.); treatment with Class IB antiarrhythmic drugs, i.e., lidocaine, tocainide, mexiletine, phenytoin; significant concomitant illness, which, in the opinion of the investigator, would interfere with the evaluation of the study medications; and treatment with topically applied medication (e.g., lidocaine/prilocaine cream, capsaicin cream, doxepin cream) 72 hours before the study. The National Cheng Kung University Hospital (NCKUH) institutional review board approved this study (BR-100-005-C).

\section{Treatment schedule}

In this study, the first visit included screening and enrollment; eligible subjects (with neuropathic cancer pain) received the study patch for three days (Fig. 1). Subsequently, the second/third visit was scheduled on the second/third day of study for endpoint evaluation. The investigator collected information on 
concurrent diseases and symptoms as well as concomitant medication and examined skin affected by the lidocaine patch. The pain intensity, including the VAS-resting score, was assessed as baseline data before patch application. The subjects were assigned to the lidocaine group for three days. The enrolled subjects applied the patch to well-defined, intact skin to cover the most painful area. Subjects were allowed to use up to three patches simultaneously, each for 12-24 hours, once to twice daily. Any observed and spontaneously reported AEs are noted. Skin inspection: A brief examination of the skin under the patches was carried out at both visits to document skin redness, blanching, or irritation. In case of any ongoing adverse events, the subject was contacted for a follow-up within one week after the trial stops to evaluate the outcome of the AEs. Any new adverse events that occurred during this period were also reported.

\section{Clinical efficacy}

The primary endpoint of the analgesic effect of the experimental patch was evaluated by assessing the VAS pain intensity. The secondary endpoints of efficacy were evaluated by the pain relief score and analgesic treatment quality. Pain intensity was evaluated using a horizontal $100 \mathrm{~mm}$ visual analog scale (VAS), which interprets the severity of pain on a scale ranging from $0=$ no pain to $10=$ the worst pain imaginable. The subject was questioned about the VAS-resting score. Furthermore, the VAS score for specific pain was recorded in parallel. The pain intensity was assessed from before the first patch was applied as the baseline (on Day 1) to after the last patch was applied (on Day 3). Pain relief score: Pain relief was assessed using a category scale consisting of 5 scores indicating the following: 0区"no" pain

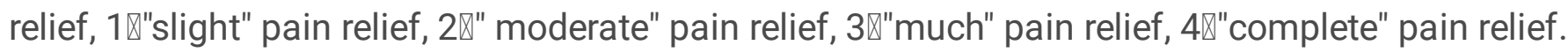
Analgesic treatment quality: The subject rated the quality of the analgesic treatment using the following five-item scale: 1 = excellent, complete pain relief, performance status reaching 100\%, no compromise of sleep and appetite; 2 = good, tolerable pain not longer than half an hour or disappearance of pain but with compromised sleep, appetite, or performance status; 3 = satisfactory, tolerable mild degree of pain, no further medication requirement; 4 = insufficient, feeling better but with pain control that was not adequate, apparent pain sensation; and 5 = poor, no improvement at all, even worse pain.

\section{Clinical safety assessments}

The safety endpoint was evaluated in terms of vital signs, skin inspections, and adverse events. The application area affected by the lidocaine patch was recorded. Vital signs consisted of systolic (SBP) and diastolic blood pressure (DBP) and the heart rate (HR), which were measured under the same conditions. A brief examination of the skin under the patches was carried out at both visits to document skin redness, blanching, or irritation. The adverse event data were listed individually by the patient and then summarized. Safety parameters such as vital signs and skin inspection were also summarized and displayed with descriptive summary statistics.

\section{Statistical analysis}


A frequency distribution was used to describe the demographic data and the distribution of each variable. The primary efficacy variable was the resting-pain intensity measurement using a horizontal $100 \mathrm{~mm}$ visual analog scale (VAS). Data analyses included descriptive and inferential statistics. Descriptive statistics, including the estimated mean and standard deviation for continuous variables, as well as the percentages and frequencies for categorical variables, were tabulated. Because of violations of the normality assumption, the Wilcoxon signed-rank test and Kruskal-Wallis test were used to compare whether the median values of the two/three groups were equal. To account for the correlation among repeated measures from the same cancer patient, a generalized estimating equation (GEE) was used to estimate the effect of covariates on the mean of the response variables. All statistical tests were 2-sided, with a P-value less than 0.05 considered to indicate statistical significance. The analyses were performed using the R 4.0.2 version software package for Windows.

\section{Results}

\section{Patient characteristics}

A total of 96 terminal cancer patients in hospice wards were referred to our studies. The median age was $58 \mathrm{Y} / 0$, and 50/46 patients were male/female. The most common types of cancer were head and neck cancer (HNSCC): 15 (15.6\%); upper gastrointestinal (UGI) cancer: 14 (14.6\%); colorectal cancer (CRC): 12 (12.5\%); genitourinary (GU) cancer: 10 (10.4\%); lung cancer: 10 (10.4\%); breast cancer: 10 (10.4\%); and hepatocellular carcinoma (HCC): 9 (9.4\%). The cancer subtypes and patient characteristics are listed in Table 1. All patients were morphine resistant, and the combination of analgesic regimens included nonsteroidal anti-inflammatory drugs (NSAIDs), acetaminophen, corticosteroids, and antidepressants. Lidocaine patches were applied on the torso (54.2\%), legs (20.8\%), head and neck (18.8\%), arms (5.2\%), and hands (1.0\%). The sensory dysfunction included allodynia (54.2\%), raised pin-prick threshold (46.9\%), and hyperalgesia (32.3\%). The dysesthesia of neuropathic pain included tingling (35.4\%), pin-pricks (31.3\%), stabbing (29.2\%), numbness (27.1\%), tightness/stretching (34.3\%), burning (20.8\%), and cutting/laceration (29.2\%). The percentages for the baseline VAS pain intensity at rest were $39(40.6 \%)$ patients with 0-3 points, 50 (52.1\%) patients with 4-7 points, and 7 (7.3\%) patients with 8-10 points. The percentages for the baseline VAS pain intensity on movement were $8(8.3 \%)$ patients with $0-3$ points, 53 $(55.2 \%)$ patients with $4-7$ points, and $35(36.5 \%)$ patients with 8-10 points.

\section{Clinical symptom relief on pain intensity}

The patients recorded their results for the three-day VAS pain intensity, pain relief score, and analgesic treatment quality. The Kruskal-Wallis test was used for pain assessment. We assessed the difference in inferring the patch's analgesic effect in patients experiencing cancer-related neuralgia under the patch. In this analysis, inferences are made with confidence levels below 95\%. The results showed a statistically significant difference in the median VAS pain intensity over three days (Fig. 2a, Kruskal-Wallis test, Pvalue $<0.0001)$. The median VAS pain intensity from Day 1 to Day 3 was 4.0 with $95 \% \mathrm{Cl}(3.3,5.0), 3.0$ $(2.5,3.5)$ and $2.5(2.0,3.0)$, respectively. The median VAS pain intensity of any two days was statistically 
significant (Wilcoxon signed-rank test, P-value $<0.0001$ ). The paired VAS pain intensity data for any two days are presented in Fig. 2b. We reported changes in the number of patients with different VAS pain intensities on any two days (Table 2). On Day 1 and Day 2, 51 patients had decreased VAS pain intensity. A total of $8.3 \%$ and $44.8 \%$ of patients had a reduction of more than 4 points and $0.5-3.5$ points, respectively. Sixteen patients had increased VAS pain intensity. A total of $14.6 \%$ and $2.1 \%$ of patients reported that their pain intensity increased by $0.5-4$ points and 5 points, respectively. There were similar results for any two days. The data are shown in Table 2.

We also used the generalized estimating equation (GEE) model for the same patient with a three-day series assessed by VAS pain intensity. The GEE estimation model shows that the VAS pain intensity on the second day decreased by approximately 0.8 points compared with the first day and that the VAS pain intensity on the third day decreased by approximately 1.4 points compared with the first day. The test results showed statistically significant differences between the VAS pain intensities on any two days (Table 3). Based on the above results, the patch's effect on pain assessed by the VAS pain intensity score was statistically significant.

\section{Effect on pain relief score}

The results showed no statistically significant difference in the median of the three-day pain relief score. Supplementary Material 1 shows that the average and median pain relief scores did not change significantly (Kruskal-Wallis test, P-value $=0.79$ ). There were no statistically significant differences in the median pain relief score for any two days as assessed by the Wilcoxon signed-rank test (P-value $=0.24$, $0.36,0.9)$.

\section{Effect on the quality of analgesic treatment}

The results showed no statistically significant difference in the median of the three-day quality of analgesic treatment. Supplementary Material 1 shows that the average and median analgesic treatment quality did not change significantly (Kruskal-Wallis test, P-value $=0.77$ ). There were no statistically significant differences in the median analgesic treatment quality for any two days as assessed by the Wilcoxon signed-rank test $(P$-value $=0.21,0.7,0.029)$.

\section{Clinical adverse events}

Minimal adverse events were noted, and these included cold sensation (6.3\%), irritation (3.1\%), itching (3.1\%), rash (3.1\%), and redness (1.0\%), as shown in Table 4.

\section{Discussion}

From the box-and-whisker plot of VAS pain intensity, we can see that the number of days of lidocaine patch use decreased the average and median VAS pain intensity. There was a statistically significant difference in the median three-day VAS pain intensity. According to the GEE estimation results, the patch's pain relief effect was statistically significant as assessed by the VAS pain intensity in the time series. The 
results of this study showed that the addition of $5 \%$ lidocaine patches could be useful in the short term for treating neuropathic cancer pain by decreasing the VAS pain intensity. However, the underlying mechanisms responsible for topical lidocaine symptom relief in patients with allodynia are not fully understood. Hans $\mathrm{G}$ et al [16] reported a prospective study including 40 patients with severe neuropathic pain due to surgical or nonsurgical trauma. Approximately $52.5 \%$ of the patients benefited from treatment with lidocaine, with the severity of their pain on a VAS diminishing at the end of the study. These results are similar to those obtained for superficial neuropathic pain following surgery.

In a placebo-controlled, crossover study, a $5 \%$ lidocaine medicated plaster effectively relieved ongoing pain and allodynia within the first eight hours, with effects lasting up to one week [17]. As in our study, we emphasized the precise selection of the patients in whom, in addition to the presence of neuropathic pain, the pain had to be well localized, superficial, and involve positive symptoms such as allodynia, raised pinprick threshold, and hyperalgesia. The severity of the pain was analyzed using a VAS, thus measuring Day 1 to Day 3. Neuropathic cancer pain cannot be treated according to the World Health Organization (WHO) recommendations because there is no evidence that such pain responds to drugs that are recommended for mild or moderate cancer pain (steps 1 and 2 of the WHO's pain relief ladder) [1]. The majority of our patients who were treated with lidocaine patches often found them practical for reducing mild and moderate neuropathic pain assessed by VAS pain intensity.

There was no statistically significant difference in the median of the three-day pain relief score or analgesic treatment quality from the box-and-whisker diagram (Supplementary Material 1). According to GEE estimates, the patch's pain relief effect was not statistically significant in terms of the pain relief score and the quality of analgesic treatment. We concluded that the patch could not be statistically supported for pain relief scores and analgesic treatment quality. Derry et al. also reviewed topical lidocaine for neuropathic pain in adults [18]. This review found no evidence from good quality randomized controlled studies to support topical lidocaine as a treatment for neuropathic pain. We did not assess the emotional dimension, quality of life, or sleep. This may constitute a limitation since the present study failed to demonstrate improvements in pain relief and quality with the lidocaine patch. We also did not find that the extent of pain interfered with daily activities.

The concept of a topical, local anesthetic patch as a therapy for allodynia has a great appeal to clinicians and patients due to a topical formulation's simplicity. The reasons for this appeal are the benefits of the patch formulation as a barrier protecting hypersensitive skin from external stimuli and the lack of clinically significant systemic absorption, drug interactions, and other serious adverse effects $[10,11]$. The present sample was not very large but included different cancer types. The results of this study showed that the investigated treatment carries no risk; nurses can apply the skin patch.

There are certain limitations to extrapolating our results; this was a non-controlled, open-label study for pain control. It is subject to a significant risk of the placebo effect and of recall bias. Unless the patients' neuropathic pain were cutaneous or superficial, a lidocaine patch was not likely to penetrate deep enough to have a pharmacological effect. Despite the restrictions in prescribing lidocaine patches, there were 
several good reasons for trying this treatment in our cancer patients: (i) the intractable nature of neuropathic pain, which is incredibly resistant to morphine, (ii) the potential benefits of the treatment in reducing VAS pain intensity, and (iii) the lack of systemic adverse side effects. These reasons led us to use this topical formulation to treat our cancer patients with neuropathic pain resistant to morphine.

\section{Conclusion}

In this study, a $5 \%$ lidocaine skin patch reduced the VAS pain intensity in patients experiencing morphineresistant neuropathic cancer pain. Such topical medication is generally useful and well tolerated.

\section{Declarations}

Funding This work was supported in part by the Ministry of Science and Technology (MOST), Taiwan under Research Grant of MOST 110-2634-F-006-014 and MOST 110-2634-F-006-020, Ministry of Health and Welfare (MOHW110-TDU-B-211-144018) and Higher Education Sprout Project, Ministry of Education to the Headquarters of University Advancement at National Cheng Kung University (NCKU).

Conflicts of interest/Competing interests None declared.

Availability of data and material The datasets used and analyzed during the current study are available from the corresponding author on reasonable request and supplementary information files of this manuscript.

Authors' contributions Conception and study design: JH Tsai, PF Su, GL Chiu, PC Lin; Development of methodology: JH Tsai, PF Su, GL Chiu, PC Lin; Acquisition of data: JH Tsai, GL Chiu, YY Chen, WS Lai, PC Lin; Statistical and computational analysis: JH Tsai , IT Liu , PF Su, YT Huang ; Writing, review, and/or revision of the manuscript: JH Tsai , IT Liu, YT Huang, PC Lin ; Study supervision: PC Lin.

Ethics approval: This study was approved by the institutional review board of NCKUH (BR-100-005-C) and conducted in accordance with the Declaration of Helsinki.

Consent to participate All participants provided written informed consent.

Consent for publication All authors agree for publication.

\section{References}

1. Devers A, Galer BS (2000) Topical lidocaine patch relieves a variety of neuropathic pain conditions: an open-label study. Clin J Pain 16(3):205-8. https://doi.org/10.1097/00002508-200009000-00005

2. Roberto A, Deandrea S, Greco MT, Corli O, Negri E, Pizzuto M, Ruggeri F (2016) Prevalence of Neuropathic Pain in Cancer Patients: Pooled Estimates From a Systematic Review of Published 
Literature and Results From a Survey Conducted in 50 Italian Palliative Care Centers. J Pain Symptom Manage 51(6):1091-1102.e4. https://doi.org/10.1016/j.jpainsymman.2015.12.336

3. Fallon MT (2013) Neuropathic pain in cancer. Br J Anaesth 111(1):105-11.

https://doi.org/10.1093/bja/aet208

4. Edwards HL, Mulvey MR, Bennett MI (2019) Cancer-Related Neuropathic Pain. Cancers (Basel) 16;11(3):373. https://doi.org/10.3390/cancers11030373

5. Colloca L, Ludman T, Bouhassira D, Baron R, Dickenson AH, Yarnitsky D, Freeman R, Truini A, Attal N, Finnerup NB, Eccleston C, Kalso E, Bennett DL, Dworkin RH, Raja SN (2017) Neuropathic pain. Nat Rev Dis Primers 16;3:17002. https://doi.org/10.1038/nrdp.2017.2

6. Martin LA, Hagen NA (1997) Neuropathic pain in cancer patients: mechanisms, syndromes, and clinical controversies. J Pain Symptom Manage 14(2):99-117. https://doi.org/10.1016/s08853924(97)00009-2

7. Devers A, Galer BS (2000) Topical lidocaine patch relieves a variety of neuropathic pain conditions: an open-label study. Clin J Pain. 2000 16(3):205-8. https://doi.org/10.1097/00002508-20000900000005

8. Demarin V, Basić-Kes V, Zavoreo I, Bosnar-Puretić M, Rotim K, Lupret V, Perić M, Ivanec Z, Fumić L, Lusić I, Aleksić-Shihabis A, Kovac B, Ivanković M, Skobić H, Maslov B, Bornstein N, Niederkorn K, Sinanović O, Rundek T (2008) Ad hoc Committee of the Croatian Society for Neurovascular Disorders; Croatian Medical Association. Recommendations for neuropathic pain treatment. Acta Clin Croat 47(3):181-91

9. Fleming JA, O'Connor BD (2009) Use of lidocaine patches for neuropathic pain in a comprehensive cancer centre. Pain Res Manag 14(5):381-8. https://doi.org/10.1155/2009/723179

10. Davies PS, Galer BS (2004) Review of lidocaine patch $5 \%$ studies in the treatment of postherpetic neuralgia. Drugs 64(9):937-47. https://doi.org/10.2165/00003495-200464090-00002

11. Singh P, Roberts MS (1994) Dermal and underlying tissue pharmacokinetics of lidocaine after topical application. J Pharm Sci 83(6):774-82.

https://doi.org/10.1002/jps.2600830604

12. Lee JS, Hobden E, Stiell IG, Wells GA (2003) Clinically important change in the visual analog scale after adequate pain control. Acad Emerg Med 10(10):1128-30. https://doi.org/10.1111/j.15532712.2003.tb00586.x

13. Angst MS, Brose WG, Dyck JB (1999) The relationship between the visual analog pain intensity and pain relief scale changes during analgesic drug studies in chronic pain patients. Anesthesiology 91(1):34-41. https://doi.org/10.1097/00000542-199907000-00009

14. Younger J, McCue R, Mackey S (2009) Pain outcomes: a brief review of instruments and techniques. Curr Pain Headache Rep 13(1):39-43. https://doi.org/10.1007/s11916-009-0009-x

15. Bennett M (2001) The LANSS Pain Scale: the Leeds assessment of neuropathic symptoms and signs. Pain 92(1-2):147-57. https://doi.org/10.1016/s0304-3959(00)00482-6 
16. Hans G, Robert D, Verhulst J, Vercauteren M (2010) Lidocaine 5\% patch for localized neuropathic pain: progress for the patient, a new approach for the physician. Clin Pharmacol 2:65-70. https://doi.org/10.2147/CPAA.S9795

17. Meier T, Wasner G, Faust M, Kuntzer T, Ochsner F, Hueppe M, Bogousslavsky J, Baron R (2003) Efficacy of lidocaine patch $5 \%$ in the treatment of focal peripheral neuropathic pain syndromes: a randomized, double-blind, placebo-controlled study. Pain 106(1-2):151-8. https://doi.org/10.1016/S0304-3959(03)00317-8

18. Derry S, Wiffen PJ, Moore RA (2014) Quinlan J. Topical lidocaine for neuropathic pain in adults. Cochrane Database Syst Rev 24;2014(7):CD010958. https://doi.org/10.1002/14651858.CD010958.pub2

\section{Tables}

Table 1. Characteristics of 96 cancer patients with neuropathic pain 


\begin{tabular}{|c|c|}
\hline Patients No. & 96 (100\%) \\
\hline \multicolumn{2}{|l|}{ Age (years) } \\
\hline Median age, yr & 58 \\
\hline Range & $20-96$ \\
\hline \multicolumn{2}{|l|}{ Performance Status } \\
\hline $1-2$ & $20(20.8 \%)$ \\
\hline $3-4$ & $76(75.2 \%)$ \\
\hline \multicolumn{2}{|l|}{ Sex } \\
\hline Male/Female & $50 / 46(58.3 \% / 41.7 \%)$ \\
\hline \multicolumn{2}{|l|}{ Diagnosis } \\
\hline HNSCC & $15(15.6 \%)$ \\
\hline UGI Cancer & $14(14.6 \%)$ \\
\hline Colorectal Cancer & $12(12.5 \%)$ \\
\hline GU Cancer & $10(10.4 \%)$ \\
\hline Lung Cancer & $10(10.4 \%)$ \\
\hline Breast Cancer & $9(9.4 \%)$ \\
\hline $\mathrm{HCC}$ & $9(9.4 \%)$ \\
\hline GYN Cancer & $6(6.3 \%)$ \\
\hline Thyroid Cancer & $6(6.3 \%)$ \\
\hline Sarcoma & $3(4.1 \%)$ \\
\hline Lymphoma & $1(1.0 \%)$ \\
\hline MUO & $1(1.0 \%)$ \\
\hline \multicolumn{2}{|c|}{ Concurrent analgesic regimen } \\
\hline Opioids & $96(100 \%)$ \\
\hline NSAID & $15(15.6 \%)$ \\
\hline Acetaminophen & $15(15.6 \%)$ \\
\hline Corticosteroid & $14(14.6 \%)$ \\
\hline Antidepressants & $7(7.3 \%)$ \\
\hline \multicolumn{2}{|l|}{ Pain at rest } \\
\hline $0-3$ & $39(40.6 \%)$ \\
\hline
\end{tabular}




\begin{tabular}{|l|l|}
\hline $4-7$ & $50(52.1 \%)$ \\
$8-10$ & $7(7.3 \%)$ \\
\hline Pain on movement & $8(8.3 \%)$ \\
$0-3$ & $53(55.2 \%)$ \\
\hline $8-7$ & $35(36.5 \%)$ \\
\hline Applied Sites & \\
\hline Torso & $52(54.2 \%)$ \\
Legs & $20(20.8 \%)$ \\
Head and Neck & $18(18.8 \%)$ \\
Arms & $5(5.2 \%)$ \\
Hands & $1(1.0 \%)$ \\
\hline Sensory Dysfunction & \\
\hline Allodynia & $52(54.2 \%)$ \\
Raised Pin-Prick Threshold & $45(46.9 \%)$ \\
\hline Hyperalgesia & $31(32.3 \%)$ \\
\hline Dysaesthesia & $28(29.2 \%)$ \\
\hline Tingling & $34(35.4 \%)$ \\
Pinprick & $30(31.3 \%)$ \\
\hline Nubbing & $28(29.2 \%)$ \\
\hline Cutting/Laceration & $26.1 \%)$ \\
\hline
\end{tabular}

Abbreviations:

HNSCC: Head and neck squamous cell carcinoma, UGI: upper gastro-intestinal, GU: genitourinary, HCC: hepatocellular carcinoma, GYN: gynecological, MUO: malignancy of unknown origin.

Table 2. Comparisons of post-treatment change scales for pain intensity 


\begin{tabular}{|ccccccc|}
\hline \multicolumn{2}{|c}{ Pain intensity with visual analog scale } \\
Decrease \\
\hline
\end{tabular}

*Decrease (Increase) was defined as a change of VAS pain intensity more than $5 \mathrm{~mm}$

**No Change was defined as a change of VAS pain intensity less than $5 \mathrm{~mm}$

Table 3. GEE model evaluation of pain intensity

\begin{tabular}{|lllll|}
\hline & Estimate & Std & Wald & P-value \\
\hline Intercept & 4.04 & 0.24 & 274.80 & $<0.0001$ \\
\hline Day2 & -0.80 & 0.19 & 17.77 & $<0.0001$ \\
\hline Day3 & -1.40 & 0.23 & 37.53 & $<0.0001$ \\
\hline
\end{tabular}

Abbreviations:

GEE: generalized estimating equation, Std: standard deviation

Table 4. Adverse events after treatment with $5 \%$ lidocaine patch

\begin{tabular}{|ll|}
\hline Adverse Events & No. (\%) \\
\hline Cold Sensation & $6(6.3 \%)$ \\
\hline Irritation & $3(3.1 \%)$ \\
\hline Itching & $3(3.1 \%)$ \\
Rash & $3(3.1 \%)$ \\
Redness & $1(1.0 \%)$ \\
\hline
\end{tabular}

\section{Figures}


Fig.1

\section{Single Arm Study}

Pre-Screen

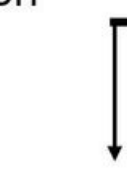

Day 1

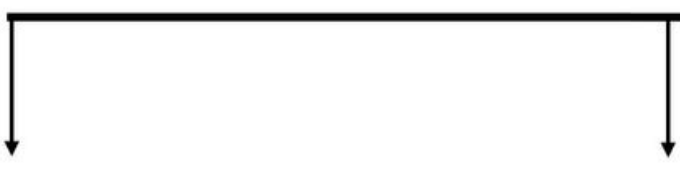

Day 2
Follow-Up

(1 Week)

Treatment : Lidocaine Patch

\begin{tabular}{llc}
\hline Visit 1 & Visit 2 & Visit 3 \\
Screen : Base-line & Evaluation & Endpoint of Evaluation
\end{tabular}

Figure 1

Treatment schedule. The first visit included screening and enrollment; eligible subjects received the study patch for three days. Subsequently, the second visit was scheduled on the second and third days of the study, and the third visit was scheduled for endpoint evaluation. 
a.
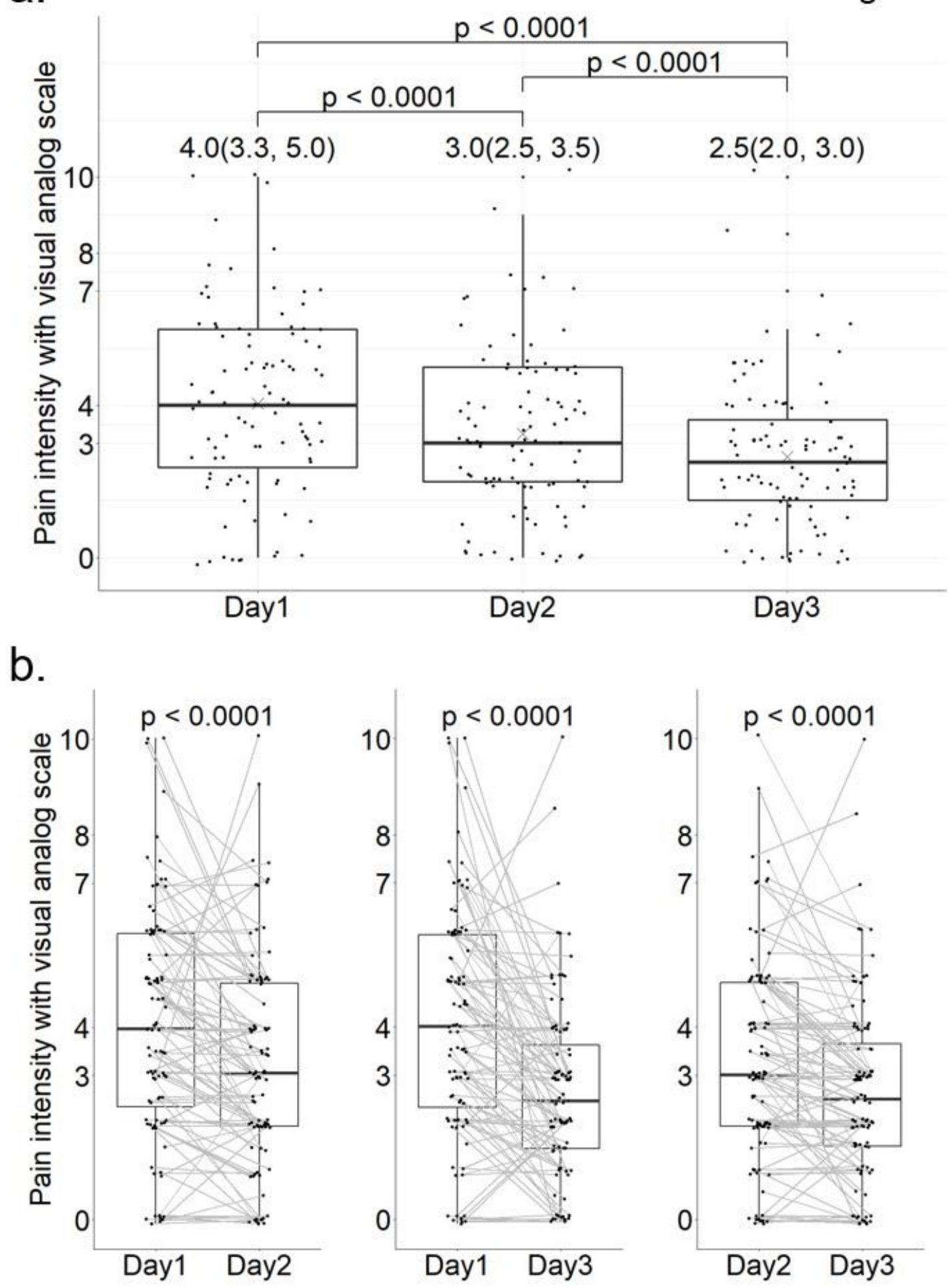

Figure 2

$a$ and $b$. Effect on pain intensity assessed by the visual analog scale Pain intensity was assessed using a horizontal $100 \mathrm{~mm}$ visual analog scale (VAS), which interprets the severity of pain on a scale ranging from $0=$ no pain to $10=$ worst pain imaginable. The pain intensity was assessed before the first patch was applied as the baseline (on Day 1) and after the last patch was used on (Day 3). 


\section{Supplementary Files}

This is a list of supplementary files associated with this preprint. Click to download.

- SupplementaryMaterial120210520.pptx 